\title{
CENTRALLY SYMMETRIC CONVEX BODIES AND SECTIONS HAVING MAXIMAL QUERMASSINTEGRALS
}

\author{
STUD. SCI. MATH. HUNGAR. 49 (2) (2012), 189-199
}

\author{
E. MAKAi, JR.* ${ }^{*}$ H. MARTini** \\ DOI: 10.1556/SScMath.49.2012.2.1197
}

\begin{abstract}
Let $d \geq 2$, and let $K \subset \mathbb{R}^{d}$ be a convex body containing the origin 0 in its interior. In a previous paper we have proved the following. The body $K$ is 0 -symmetric if and only if the following holds. For each $\omega \in S^{d-1}$, we have that the $(d-1)$-volume of the intersection of $K$ and an arbitrary hyperplane, with normal $\omega$, attains its maximum if the hyperplane contains 0 . An analogous theorem, for 1-dimensional sections and 1-volumes, has been proved long ago by Hammer $([\mathrm{H}])$. In this paper we deal with the $((d-2)$-dimensional $)$ surface area, or with lower dimensional quermassintegrals of these intersections, and prove an analogous, but local theorem, for small $C^{2}$-perturbations, or $C^{3}$-perturbations of the Euclidean unit ball, respectively.
\end{abstract}

\section{INTRODUCTION}

Let $d \geq 2$, and let $K \subset \mathbb{R}^{d}$ be a centered, i.e., 0 -symmetric convex body. We have observed in [MMÓ], Problem 3.10, that by the Aleksandroff-Fenchel inequalities (cf., e.g., [S]) we have the following statement. Let $0 \leq l \leq d-2$ be an integer, let $\omega \in S^{d-1}$, let $t \in \mathbb{R}$, and let $\omega^{\perp}$ be the orthocomplement of $\omega$ in $\mathbb{R}^{d}$. Then the quermassintegrals

$$
W_{l}\left[\left(K \cap\left(\omega^{\perp}+t \omega\right)\right)-t \omega\right],
$$

considered in $\omega^{\perp}$, attain their maxima for $t=0$. In the same Problem 3.10, we have posed the question, whether the converse implication holds. For $l=0$, i.e., for the case of $(d-1)$-volume, we proved this converse implication, cf. [MMÓ], Corollary 3.2.

In this paper, we deal with the cases $1 \leq l \leq d-2$, and prove an infinitesimal variant of the converse implication, for small $C^{2}$-perturbations of the Euclidean unit ball for $l=1$, and for small $C^{3}$-perturbations of the Euclidean unit ball for $2 \leq l \leq d-2$.

1991 Mathematics Subject Classification. 2000 Mathematics Subject Classification. Primary: 52A20. Secondary: 52A39.

Key words and phrases. Convex bodies, sections, quermassintegrals, 0-symmetry.

* Research (partially) supported by Hungarian National Foundation for Scientific Research, grant nos. K68398, K75016, K81146, and DFG-GZ MA 1329/26-1.

** Research partially supported by DFG. 


\section{Preliminaries}

We write $\mathbb{R}^{d}$ for the $d$-dimensional Euclidean space, and $S^{d-1}$ for its unit sphere, where $d \geq 2$. The origin is denoted by 0 . We write $W_{i}$ for the $(k-i)$-dimensional quermassintegrals of convex bodies in affine $k$-subspaces of $\mathbb{R}^{d}([\mathrm{BF}],[\mathrm{S}])$.

Basically we use the notations of [MMÓ]. Variable points of $S^{d-1}$ are denoted by $\omega, \xi, \eta$. We use polar coordinates on $S^{d-1}$. That is, for some $\xi \in S^{d-1}$, that we consider as the north pole, and for $\omega \in S^{d-1}$, we write

$$
\omega=\xi \sin \psi+\eta \cos \psi, \text { where } \eta \in \xi^{\perp} \cap S^{d-1} \text {, and }-\pi / 2 \leq \psi \leq \pi / 2 \text {. }
$$

Thus, $\xi^{\perp} \cap S^{d-1}$ is the equator and $\psi$ is the geographic latitude, that will be more convenient to us than the customarily used $\varphi=\pi / 2-\psi$. Then we write

$$
\omega=(\eta, \psi)
$$

In particular,

$$
(\eta, 0)=\eta
$$

A function $f:=S^{d-1} \rightarrow \mathbb{R}$ is even, or odd, if, for all $\omega \in S^{d-1}$, we have $f(-\omega)=$ $f(\omega)$, or $f(-\omega)=-f(\omega)$, respectively.

In $\mathbb{R}^{d}$ we will use polar coordinates $\omega, \varrho$, with $\omega \in S^{d-1}$, and $\varrho \in[0, \infty)$ (i.e., the point $\omega \varrho \in \mathbb{R}^{d}$ has polar coordinates $\left.\omega, \varrho\right)$. Also, for fixed $\xi \in S^{d-1}$, we will use cylindrical coordinates $\eta, r, t$, with $\eta \in \xi^{\perp} \cap S^{d-1}$, and $r \in[0, \infty)$ (together polar coordinates in $\xi^{\perp}$ ), and $t \in \mathbb{R}$. Here, for $x \in \mathbb{R}^{d}$, we have

$$
t=\langle x, \xi\rangle, \text { and } x=r \eta+t \xi \text {. }
$$

For $x \in \mathrm{bd} K$, we will also write, in cylindrical coordinates,

$$
x=r(\xi, \eta, t) \eta+t \xi
$$

where the first variable of $r$ refers to $\xi$, and the last variable means that we consider the radial function of the intersection $K \cap\left(\xi^{\perp}+t \xi\right)$, with respect to the "origin" $t \xi$.

We have, for $x \in \mathbb{R}^{d}$, that

$$
\varrho \cos \psi=r \text {, and } \varrho \sin \psi=t \text {. }
$$

Differentiating these formulas with respect to $\psi$, and then setting $\psi=0$, we obtain

$$
\left.\frac{\partial r}{\partial \psi}\right|_{\psi=0}=\left.\frac{\partial \varrho}{\partial \psi}\right|_{\psi=0}, \text { and }\left.\frac{\partial t}{\partial \psi}\right|_{\psi=0}=\left.\varrho\right|_{\psi=0}
$$

For terms undefined in this paper, cf., e.g., [BF], and [S].

\section{ThEOREM}


Theorem. Let $d \geq 3$ and $2 \leq k \leq d-1$ be integers, and let $\lambda_{0} \in(0, \infty)$. Suppose that for each $\lambda \in\left[0, \lambda_{0}\right]$, we have that $K^{\lambda}$ is a convex body in $\mathbb{R}^{d}$ with radial function $\varrho^{\lambda}(\omega)$, for $\omega \in S^{d-1}$. Let $\varrho^{0}(\omega) \equiv 1$, and let $\varrho^{\lambda}(\omega)$ be a $C^{2}$-function of $(\lambda, \omega) \in\left[0, \lambda_{0}\right] \times S^{d-1}$. Assume that for each $\lambda \in\left[0, \lambda_{0}\right]$, for any linear $k$-subspace $L_{k} \subset \mathbb{R}^{d}$, the function $y \mapsto W_{1}\left(K^{\lambda} \cap\left(L_{k}+y\right)\right)$ has a maximum at $y=0$. Then

$$
\left.\frac{\partial \varrho^{\lambda}}{\partial \lambda}(\omega)\right|_{\lambda=0}
$$

is an even function of $\omega$. If $2 \leq l \leq k-1$, and $\varrho^{\lambda}(\omega)$ is a $C^{3}$-function of $(\lambda, \omega) \in$ $\left[0, \lambda_{0}\right] \times S^{d-1}$, and we replace in the above hypothesis $W_{1}$ by $W_{l}$, then the same conclusion holds.

Clearly, we could have written, in the hypothesis of the theorem, that $K^{\lambda}$ is a star body, since, by the other assumptions, $K^{\lambda}$ is a convex body with positive Gauss curvature for each $\lambda \in\left[0, \lambda_{0}\right]$ (after possibly decreasing $\lambda_{0}$ ).

We observe that for the case $k=1$, and for the case $l=0$, we have the theorems cited in the abstract, cf. [H], Theorem 1, and [MMÓ], Corollary 3.2. These assert that, in this case, actually each $K^{\lambda}$ is centered, which is of course a stronger statement than the statement of the theorem of this paper. There is still one quermassintegral, namely $W_{k}$. However, this is, independently of its argument, equal to the volume of the unit ball in $\mathbb{R}^{k}$, so, in this case the hypotheses of our theorem do not imply anything.

\section{ProOF}

We begin with the following

Proposition. ([MMÓ], Theorem 3.8). Let $f: S^{d-1} \rightarrow \mathbb{R}$ be a $C^{1}$-function (or, more generally, a Lipschitz function). Further, let, for each $\xi \in S^{d-1}$ (or, more generally, for almost all $\left.\xi \in S^{d-1}\right)$, the equality

$$
\int_{\xi^{\perp} \cap S^{d-1}} \frac{\partial f}{\partial \psi}(\eta, 0) d \eta=0
$$

hold. Then $f$ is an even function.

Proof of the Theorem. As in the proof of Theorem 3.1 of [MMÓ], we may suppose $k=d-1$. In fact, for any linear $(k+1)$-subspace $L_{k+1}$ of $\mathbb{R}^{d}$, we have that $K^{\lambda} \cap L_{k+1}$ also satisfy the hypotheses of the theorem. Furthermore, if for each $L_{k+1}$, the restriction of the function

$$
\left.\frac{\partial \varrho^{\lambda}}{\partial \lambda}(\omega)\right|_{\lambda=0}
$$

to $L_{k+1}$ is even, then also this function itself is even. So, from now on, let $k=d-1$.

Let $t_{0} \in(0, \infty)$ be so small that the closed ball about 0 , of radius $t_{0}$, is contained in each $K^{\lambda}$, where $\lambda \in\left[0, \lambda_{0}\right]$ (possibly decreasing $\lambda_{0}$ ). From now on, let 
$t \in\left(-t_{0}, t_{0}\right)$. This implies that $K \cap\left(\xi^{\perp}+t \xi\right)$ is a $((d-1)$-dimensional $)$ convex body in $\xi^{\perp}+t \xi$.

1. First we treat the case $l=1$.

Let us fix a point $\xi \in S^{d-1}$, that we consider as the north pole. Let $S^{\lambda}(\xi, t)$ denote the $((d-2)$-dimensional $)$ surface area of $K^{\lambda} \cap\left(\xi^{\perp}+t \xi\right)$, considered as a $\left((d-1)\right.$-dimensional) convex body in $\xi^{\perp}+t \xi$. We have

$$
S^{\lambda}(\xi, t)=\int_{\xi^{\perp} \cap S^{d-1}} d S^{\lambda}(\xi, t)=\int_{\xi^{\perp} \cap S^{d-1}} r^{\lambda}(\xi, \eta, t)^{d-2} \frac{1}{\left\langle\eta, n^{\lambda}(\eta, t)\right\rangle} d \eta
$$

where $r^{\lambda}(\xi, \eta, t)$ is the radial function of $K^{\lambda} \cap\left(\xi^{\perp}+t \xi\right)$, with respect to the "origin" $t \xi$, and $n^{\lambda}(\eta, t) \in \xi^{\perp} \cap S^{d-1}$ is the outer normal unit vector of the surface element $d S^{\lambda}(\xi, t)$ at $\eta \in \xi^{\perp} \cap S^{d-1}$, taken in $\xi^{\perp}+t \xi$.

From now on, we consider $\lambda \in\left[0, \lambda_{0}\right]$ as fixed, and drop the upper indices $\lambda$. Also, to simplify the formulas, we omit those variables of our functions, whose omission does not lead to misunderstanding.

We determine

$$
\left.\frac{\partial}{\partial t} S(\xi, t)\right|_{t=0}
$$

that equals 0 by the hypothesis of the theorem. We may differentiate under the integral sign. We have

$$
\left\{\begin{array}{l}
(\partial / \partial t)\left(r^{d-2} /\langle\eta, n(\eta)\rangle\right)= \\
(d-2) r^{d-3}(\partial r / \partial t) /\langle\eta, n(\eta)\rangle-r^{d-2}[(\partial / \partial t)\langle\eta, n(\eta)\rangle] /\langle\eta, n(\eta)\rangle^{2}
\end{array}\right.
$$

and we have to evaluate this at $t=0$.

Letting $t=0$, i.e., by $(1), \psi=0$, we have by $(2) \partial t / \partial \psi=\varrho$, hence

$$
\frac{\partial}{\partial t}=\frac{\partial \psi}{\partial t} \frac{\partial}{\partial \psi}=\frac{1}{\varrho} \frac{\partial}{\partial \psi}
$$

Therefore, (4) equals

$$
(d-2) r^{d-3} \frac{1}{\varrho} \frac{\partial r}{\partial \psi} \frac{1}{\langle\eta, n(\eta)\rangle}-r^{d-2} \frac{1}{\langle\eta, n(\eta)\rangle^{2}} \frac{1}{\varrho} \frac{\partial}{\partial \psi}\langle\eta, n(\eta)\rangle .
$$

Here the first term is, using $r=\varrho$ (cf. (1)),

$$
(d-2) \varrho^{d-4} \frac{\partial \varrho}{\partial \psi} \frac{1}{\langle\eta, n(\eta)\rangle}
$$

and the second term is

$$
-\varrho^{d-3} \frac{1}{\langle\eta, n(\eta)\rangle^{2}} \frac{\partial}{\partial \psi}\langle n, n(\eta)\rangle
$$


Now it will be convenient to write $\varrho=: 1+\varepsilon$, where $\varepsilon: S^{d-1} \rightarrow \mathbb{R}$ is a $C^{2}$ function, of $C^{2}$-norm tending to 0 for $\lambda \rightarrow 0$. We calculate (5) and (6), till terms of degree 1 in $\varepsilon$, but neglecting terms of degree at least 2 in $\varepsilon$.

Then (5) becomes

$$
(d-2)(1+\varepsilon)^{d-4} \frac{\partial \varepsilon}{\partial \psi} \frac{1}{\langle\eta, n(\eta)\rangle} .
$$

Here, because of the third factor, we may write $\varepsilon=0$ in the second and fourth factors, getting

$$
(d-2) \frac{\partial \varepsilon}{\partial \psi} .
$$

On the other hand, (6) contains $(\partial / \partial \psi)\langle\eta, n(\eta)\rangle$ as a factor. We are going to show that this is an expression of second order in $\varepsilon$. We have

$$
n(\eta)=\frac{\left(1,-\partial \varepsilon / \partial x_{1}, \ldots,-\partial \varepsilon / \partial x_{d-1}\right)}{\sqrt{1+\left(\partial \varepsilon / \partial x_{1}\right)^{2}+\cdots+\left(\partial \varepsilon / \partial x_{d-1}\right)^{2}}},
$$

where $x_{1}, \ldots, x_{d-1}$ are the coordinates on $S^{d-1}$, in a neighbourhood of $\eta$, given by the inverse of the exponential map at $\eta \in S^{d-1}$. (The exponential map maps vectors $u$, in a neighbourhood of the origin $\eta$ of the tangent plane of $S^{d-1}$ at $\eta$, to the point $\omega \in S^{d-1}$ of the geodesic on $S^{d-1}$, starting from $\eta$, in the direction of $u$, with $\omega$ being at a geodesic distance $\|u\|$ from $\eta$.) Therefore,

$$
\langle\eta, n(\eta)\rangle=\frac{1}{\sqrt{1+\left(\partial \varepsilon / \partial x_{1}\right)^{2}+\cdots+\left(\partial \varepsilon / \partial x_{d-1}\right)^{2}}} .
$$

Clearly, it is enough to show that, e.g.,

$$
\frac{\partial}{\partial x_{1}} \frac{1}{\sqrt{1+\left(\partial \varepsilon / \partial x_{1}\right)^{2}+\cdots+\left(\partial \varepsilon / \partial x_{d-1}\right)^{2}}}
$$

is of second degree of smallness in $\varepsilon$. However, (8) equals

$$
-\frac{\left(\partial \varepsilon / \partial x_{1}\right)\left(\partial^{2} \varepsilon / \partial x_{1}^{2}\right)+\cdots+\left(\partial \varepsilon / \partial x_{d-1}\right)\left(\partial^{2} \varepsilon /\left(\partial x_{d-1} \partial x_{1}\right)\right)}{\left(1+\left(\partial \varepsilon / \partial x_{1}\right)^{2}+\cdots+\left(\partial \varepsilon / \partial x_{d-1}\right)^{2}\right)^{3 / 2}},
$$

and so our claim is shown.

Altogether, by (3) and (4), and, on the one hand, by (5) and (7), on the other hand, by (6) and (9), we have that $\left.((\partial / \partial t) S(\xi, t))\right|_{t=0}$ is, till terms of degree 1 in $\varepsilon$,

$$
(d-2) \int_{\xi^{\perp} \cap S^{d-1}} \frac{\partial \varepsilon}{\partial \psi} d \eta
$$


Since, for each $\xi \in S^{d-1}$, (10) equals 0 , the Proposition implies that $\varepsilon$ is even. (Recall that, by hypothesis, $d \geq 3$.) Returning to the original notations,

$$
\left.\frac{\partial \varrho^{\lambda}}{\partial \lambda}(\omega)\right|_{\lambda=0}
$$

is an even function of $\omega$.

2. Now we treat the case $2 \leq l \leq d-2$.

Actually, we will allow $1 \leq l \leq d-1$. Of course, as stated after the theorem, for $l=d-1$ the statement of the theorem does not hold. However, we will need this case for our formulas.

We have, for $1 \leq l \leq d-1$, that

$$
\left\{\begin{array}{l}
W_{l}^{\lambda}(\xi, t):=W_{l}\left(K \cap\left(\xi^{\perp}+t \xi\right)\right)= \\
(1 /(d-1)) \int_{\operatorname{bd}\left(K \cap\left(\xi^{\perp}+t \xi\right)\right)} H_{l-1}(\xi, t) d S^{\lambda}(\xi, t) .
\end{array}\right.
$$

Here $H_{l-1}(\xi, t)$ is $\left(\begin{array}{c}d-2 \\ l-1\end{array}\right)^{-1}$ times the $(l-1)$ 'st elementary symmetric function of the $d-2$ principal curvatures $\kappa_{1}(\xi, t), \ldots, \kappa_{d-2}(\xi, t)$ of bd $\left(K \cap\left(\xi^{\perp}+t \xi\right)\right)$. Cf., e.g., [S], p. 291.

We write $\kappa_{i}(\xi, t)=: 1+\delta_{i}(\xi, t)$, where $\delta_{i}$ is of first order with respect to the $C^{2}$-norm of $\varepsilon=\varrho-1$.

Letting

$$
\left\{\begin{array}{l}
P:=\left(\begin{array}{c}
d-2 \\
l-1
\end{array}\right)^{-1} \sum_{1 \leq i_{1}<\cdots<i_{l-1} \leq d-2}\left(1+\delta_{i_{1}}\right) \ldots\left(1+\delta_{i_{l-1}}\right) \\
-\left(\begin{array}{c}
d-2 \\
l-1
\end{array}\right)^{-1} \sum_{1 \leq i_{1}<\cdots<i_{l-1} \leq d-2}\left(1+\delta_{i_{1}}+\cdots+\delta_{i_{l-1}}\right) \\
=\left(\begin{array}{c}
d-2 \\
l-1
\end{array}\right)^{-1} \sum_{1 \leq i_{1}<\cdots<i_{l-1} \leq d-2}\left(1+\delta_{i_{1}}\right) \ldots\left(1+\delta_{i_{l-1}}\right) \\
-1-((l-1) /(d-2)) \sum_{i=1}^{d-2} \delta_{i}
\end{array}\right.
$$

we have that $P$ is a linear combination with constant coefficients, of the elementary symmetric functions of the $\delta_{i}$ 's, of degrees 2 to $l-1$. Therefore, $\partial P / \partial t$ is a sum, whose summands are products of some $\partial \delta_{i} / \partial t$, and at least one further $\delta_{j}$. Here $\partial \delta_{i} / \partial t$ is bounded by the $C^{3}$-assumption, and the $\delta_{j}$ 's are of first order with respect to the $C^{2}$-norm of $\varepsilon$. Hence, when calculating the derivative of (11), with respect to $t$, at $t=0$, we can neglect $\partial P / \partial t$. Hence, we may replace in (11) $H_{l-1}$ by

$$
1+\frac{l-1}{d-2} \sum_{i=0}^{d-2} \delta_{i}
$$

and this replacement will not affect the calculation of the derivative of (11), with respect to $t$, at $t=0$. 
We turn to the calculation of the derivative of (11), with respect to $t$, at $t=0$, which has to be 0 . As mentioned above, this equals

$$
\left\{\begin{array}{l}
(1 /(d-1))(\partial / \partial t) \int_{\operatorname{bd}\left(K \cap\left(\xi^{\perp}+t \xi\right)\right)} d S^{\lambda}(\xi, t)+ \\
(l-1) /((d-1)(d-2))(\partial / \partial t) \int_{\operatorname{bd}\left(K \cap\left(\xi^{\perp}+t \xi\right)\right)}\left(\sum_{i=0}^{d-2} \delta_{i}\right) d S^{\lambda}(\xi, t) .
\end{array}\right.
$$

Here the first summand is, by $\mathbf{1}$,

$$
\frac{d-2}{d-1} \int_{\xi^{\perp} \cap S^{d-1}} \frac{\partial \varepsilon}{\partial \psi} d \eta .
$$

We are going to determine the second summand. For this, put $l=d-1$. Then, as already mentioned, (11) is constant, hence (13) equals 0. From this we have

$$
\frac{\partial}{\partial t} \int_{\operatorname{bd}\left(K \cap\left(\xi^{\perp}+t \xi\right)\right)}\left(\sum_{i=0}^{d-2} \delta_{i}\right) d S^{\lambda}(\xi, t)=-(d-2) \int_{\xi^{\perp} \cap S^{d-1}} \frac{\partial \varepsilon}{\partial \psi} d \eta .
$$

Hence, for all $l=1, \ldots, d-1$, we have that (13) further equals

$$
\frac{d-1-l}{d-l} \int_{\xi^{\perp} \cap S^{d-1}} \frac{\partial \varepsilon}{\partial \psi} d \eta,
$$

which equals 0 . By the hypothesis of the theorem, we have $l \leq d-2$, hence

$$
\int_{\xi^{\perp} \cap S^{d-1}} \frac{\partial \varepsilon}{\partial \psi} d \eta=0
$$

for each $\xi \in S^{d-1}$. As in $\mathbf{1}$, this implies that

$$
\left.\frac{\partial \varrho^{\lambda}}{\partial \lambda}(\omega)\right|_{\lambda=0}
$$

is an even function of $\omega$.

\section{REMARK}

Remark. Let $\lambda_{0} \in(0, \infty)$. Let $K^{0} \subset \mathbb{R}^{d}$ be a centered convex body, further suppose that for each $\lambda \in\left(0, \lambda_{0}\right]$, we have that $K^{\lambda}$ is a convex body in $\mathbb{R}^{d}$, with radial functions $\varrho^{\lambda}$, for $\lambda \in\{0\} \cup\left(0, \lambda_{0}\right]=\left[0, \lambda_{0}\right]$. Moreover, let $\varrho^{\lambda}(\omega)$ be a $C^{2}$ function of $(\lambda, \omega) \in\left[0, \lambda_{0}\right] \times S^{d-1}$. We may ask whether some analogue of our 
theorem holds. That is, suppose that for each $\lambda \in\left[0, \lambda_{0}\right]$, and each linear $(d-1)$ subspace $L_{d-1} \subset \mathbb{R}^{d}$, the function $y \mapsto W_{l}\left(K^{\lambda} \cap\left(L_{d-1}+y\right)\right)$ has a maximum at $y=0$. Then we may pose the question: is

$$
\left.\frac{\partial \varrho^{\lambda}}{\partial \lambda}(\omega)\right|_{\lambda=0}
$$

an even function of $\omega$ ? However, we will show that this question, even in the simplest unsolved case, i.e., for $d=3$, and for $W_{1}$, is untreatable by our present methods.

For $d-1=2$ we can use, for the calculation of the perimeter of $K^{\lambda} \cap\left(\xi^{\perp}+t \xi\right)$, the simpler formula $d s^{2}=d r^{2}+r^{2} d \eta^{2}$. Then the equality

$$
\left.\left(\frac{\partial}{\partial t} W_{1}\left[\left(K \cap\left(\xi^{\perp}+t \xi\right)\right)-t \xi\right]\right)\right|_{t=0}=0
$$

can be rewritten as

$$
\int_{S^{1}} \frac{1}{\sqrt{\varrho^{2}+(\partial \varrho / \partial \eta)^{2}}}\left(\frac{\partial \varrho}{\partial \psi}+\frac{1}{\varrho} \frac{\partial \varrho}{\partial \eta} \frac{\partial^{2} \varrho}{\partial \eta \partial \psi}\right) d \eta=0 .
$$

Let us write $\varrho^{\lambda}=\varrho^{0}+\varepsilon$. We retain in (15) the terms at most linear in $\varepsilon$, and investigate this situation. Clearly, the terms of degree 0 in $\varepsilon$ together give the integral, on $S^{1}$, of an odd function, i.e., 0. Now we investigate the terms of degree 1 in $\varepsilon$, in the expression under the integral sign in (15). These are the following:

$$
\left\{\begin{array}{l}
{\left[-\left[\left(\varrho^{0}\right)^{2}+\left(\partial \varrho^{0} / \partial \eta\right)^{2}\right]^{-1 / 2}\left(\varrho^{0}\right)^{-2}\left(\partial \varrho^{0} / \partial \eta\right) \partial^{2} \varrho^{0} /(\partial \eta \partial \psi)\right.} \\
\left.-\varrho^{0}\left(\left(\varrho^{0}\right)^{2}+\left(\partial \varrho^{0} / \partial \eta\right)^{2}\right)^{-3 / 2}\left(\partial \varrho^{0} / \partial \psi+\left(\varrho^{0}\right)^{-1}\left(\partial \varrho^{0} / \partial \eta\right) \partial^{2} \varrho^{0} /(\partial \eta \partial \psi)\right)\right] \varepsilon \\
+\left[\left(\varrho^{0}\right)^{2}+\left(\partial \varrho^{0} / \partial \eta\right)^{2}\right]^{-1 / 2}(\partial \varepsilon / \partial \psi)+\left[\left[\left(\varrho^{0}\right)^{2}+\left(\partial \varrho^{0} / \partial \eta\right)^{2}\right]^{-1 / 2}\left(\varrho^{0}\right)^{-1} \partial^{2} \varrho^{0} /\right. \\
(\partial \eta \partial \psi)-\left(\partial \varrho^{0} / \partial \eta\right)\left[\left(\varrho^{0}\right)^{2}+\left(\partial \varrho^{0} / \partial \eta\right)^{2}\right]^{-3 / 2}\left(\partial \varrho^{0} / \partial \psi+\left(\varrho^{0}\right)^{-1}\left(\partial \varrho^{0} / \partial \eta\right) \partial^{2} \varrho^{0} /\right. \\
(\partial \eta \partial \psi))] \partial \varepsilon / \partial \eta+\left[\left(\varrho^{0}\right)^{2}+\left(\partial \varrho^{0} / \partial \eta\right)^{2}\right]^{-1 / 2}\left(\varrho^{0}\right)^{-1}\left(\partial \varrho^{0} / \partial \eta\right) \partial^{2} \varepsilon /(\partial \eta \partial \psi) \\
=: A \varepsilon+B \partial \varepsilon / \partial \psi+C \partial \varepsilon / \partial \eta+D \partial^{2} \varepsilon /(\partial \eta \partial \psi) .
\end{array}\right.
$$

Now, let us suppose that $\varrho^{\lambda}(\omega)$ is a $C^{3}$ function of $(\lambda, \omega) \in\left[0, \lambda_{0}\right] \times S^{2}$. Then, retaining in (15) the terms at most linear in $\varepsilon$, (15) becomes, by integration by parts,

$$
\int_{S^{1}}\left[\left(A-\frac{\partial C}{\partial \eta}\right) \varepsilon+\left(B-\frac{\partial D}{\partial \eta}\right) \frac{\partial \varepsilon}{\partial \psi}\right] d \eta=0
$$

(We do not give the coefficients in this formula more explicitly.) Of course, the left hand side of (17) is a continuous linear operator in $\varepsilon$, for the $C^{1}$-topology. But its solution (e.g., that the solutions among the $C^{1}$-functions would be just the even $C^{1}$-functions) seems to be untreatable by our methods. 


\section{REFERENCES}

[BF] T. Bonnesen, W. Fenchel, Theorie der konvexen Körper. Berichtigter Reprint, Springer, Berlin-New York, 1974. MR 49\#9736.

[H] P. C. Hammer, Diameters of convex bodies, Proc. Amer. Math. Soc. 5 (1954), 304-306. MR 15,819b.

[MMÓ] E. Makai, Jr., H. Martini, T. Ódor, Maximal sections and centrally symmetric bodies, Mathematika 47 (2000), 19-30. MR 2003e:52005.

[S] R. Schneider, Convex Bodies: The Brunn-Minkowski Theory, Encyclopedia of Mathematics and its Applications 44, Cambridge University Press, Cambridge, 1993. MR 94d:52007.

E. MAKAI, JR.*,

Alfréd Rényi Mathematical Institute, Hungarian Academy of Sciences, H-1364 Budapest, Pf. 127, HUNGARY, http://www.renyi.mta.hu/ makai

H. MARTini**,

Technische Universität Chemnitz, Fakultät für Mathematik, D-09107 Chemnitz, GERMANY, WWW.TU-CHEMNITZ.DE/MATHEMATIK/GEOMETRIE

E-mail address: makai.endre@renyi.mta.hu, martini@mathematik.tu-chemnitz.de 